\title{
Proteomic analysis of calcified abdominal and thoracic aortic aneurysms
}

\author{
KEN-ICHI MATSUMOTO ${ }^{1}$, TOMOKO MANIWA ${ }^{1}$, TETSUYA TANAKA ${ }^{2}$, \\ KAZUMI SATOH $^{1}$, HIDEKI OKUNISHI ${ }^{2}$ and TEIJI ODA ${ }^{3}$ \\ ${ }^{1}$ Department of Biosignaling and Radioisotope Experiment, Center for Integrated Research in Science, Shimane \\ University, Izumo; ${ }^{2}$ Department of Pharmacology and ${ }^{3}$ Division of Cardiovascular and Thoracic \\ Surgery, Department of Surgery, Shimane University School of Medicine, Izumo, Japan
}

Received February 20, 2012; Accepted April 12, 2012

DOI: $10.3892 / \mathrm{ijmm} .2012 .985$

\begin{abstract}
Aortic aneurysm is a complex multifactorial disease with genetic and environmental risk factors. It is often accompanied by aortic calcification. Here, to uncover proteins that are significantly changed in calcified abdominal aortic aneurysms (CAAs) and calcified thoracic aortic aneurysms (CTAs) compared with those in adjacent normal aorta tissues, comprehensive analysis of differentially expressed proteins in their tissues was performed by a quantitative proteomic approach with iTRAQ labeling in combination with nanoLC-MALDITOF/TOF-MS/MS followed by ProteinPilot analysis. The proteomic analysis revealed 138 and 134 proteins differentially expressed in CAAs and CTAs in contrast to neighboring normal aorta tissues with high confidence, respectively. Significantly increased expression ( $\geq 1.3$-fold) was found in 41 and 28 proteins, whereas decreased expression $(<0.77$-fold $)$ was found in 4 and 60 proteins in CAAs and CTAs, respectively. Among them, we identified already known proteins involved in aneurysm formation and vascular calcification, such as type I and III collagen, matrix Gla protein, and $\alpha-2-\mathrm{HS}$-glycoprotein in CAAs and fibrinogen $\alpha, \beta$ and $\gamma$ chains and $\alpha-2-H S-$ glycoprotein in CTAs with increased expression and mimecan in CAAs and fibulin-5 in CTAs with decreased expression. Based on the Panther pathway and Genesis clustering analyses, some of the proteins could be linked to corresponding biochemical pathways, such as the integrin signaling pathway with increased expression in CAAs, the blood coagulation pathway with increased expression in CTAs, and the inflammation mediated by chemokine and cytokine signaling pathway and the glycolysis pathway with decreased expression
\end{abstract}

Correspondence to: Dr Ken-ichi Matsumoto, Department of Biosignaling and Radioisotope Experiment, Center for Integrated Research in Science, Shimane University, 89-1 Enya-cho, Izumo 693-8501, Japan

E-mail: matumoto@med.shimane-u.ac.jp

Key words: calcified abdominal aortic aneurysm, calcified thoracic aortic aneurysm, isobaric tag for absolute and relative quantitation, proteomics in CTAs. Interestingly, it was found by clustering analysis that samples from CAAs of patients with both CAAs and CTAs were clustered outside the samples of patients with CAAs and were clustered with samples of patients with CTAs. Our results provide a comprehensive patient-based proteomic analysis for the identification of potential biomarkers for CAAs and CTAs.

\section{Introduction}

Aortic aneurysm is a severe cardiovascular disease with high mortality and morbidity rates. It is associated with advanced age, male gender, cigarette smoking, atherosclerosis, hypertension, family history, and genetic predisposition (1). Aortic aneurysms most frequently arise in the infrarenal abdominal aorta [so-called abdominal aortic aneurysm (AAA)] and the descending or ascending portion of the thoracic aorta [so-called thoracic aortic aneurysm (TAA)].

Previous studies have provided evidence for involvement of proteolysis and the immune system in aortic aneurysm formation and progression at the molecular level $(2,3)$. Histologically, fragmentation and degeneration of elastin and collagen are generally observed in the media in the aneurysm wall. Numerous molecular mediators and extracellular matrixdegrading proteinases participate in the progression of AAAs. Matrix metalloproteinases (MMPs) and their antagonists, tissue inhibitors of metalloproteinases (TIMPs), are instrumental in aneurysm formation and progression. Members of the gelatinase subclass, MMP-2 and MMP-9, have been studied with regard to aneurysm formation (2). Growth and rupture of AAAs result from increased collagen turnover by increased type I collagen degradation within AAAs (4). Other causes of aortic wall weakening are apoptosis of vascular smooth muscle cells (VSMCs) and transmural infiltration of lymphocytes and macrophages within AAA aortas providing the stimulation for a chronic inflammatory response (5). An association of atherosclerotic lesions with the formation of AAAs in the intima of the aorta has also been proposed, although it is still controversial. Supporting evidence for the association of AAAs with atherosclerosis includes similar aortic regions being prone to both diseases (6). On the other hand, there are several lines of evidence suggesting that atherosclerosis is not a prerequisite for AAA formation (7). 
The prevalence of TAAs is only about one-third of that of AAAs (3). Structural heterogeneity of the aorta has been shown between the thoracic and abdominal aortic regions (8). The structural heterogeneity of thoracic and abdominal aortas may contribute to the differences observed in the pathogenesis of AAAs and TAAs. The thoracic aorta has a thinner intima, thicker media, and more medial lamellar units than those of the abdominal aorta. In addition, the thoracic aorta has significantly higher elastin and collagen contents and a lower collagen-toelastin ratio than those of the abdominal aorta. In contrast to the abdominal region, atherosclerosis rarely presents in the thoracic region. Medial degeneration is qualitatively and quantitatively much greater in patients with TAAs than in patients with AAAs. Lesauskaite et al (9) demonstrated that MMP-2 and MMP-9 activities were increased at portions of medial degeneration in aortas from patients with TAAs. Furthermore, $\sim 20 \%$ of TAAs are attributed to some genetic syndromes (10).

In many cases, aortic aneurysms are accompanied by vascular calcification. Calcified arteries lose their elasticity, resulting in exacerbation of ischemia, and increase in cardiac afterload. Vascular calcification occurs at two histological sites of arteries, the intima and the media. Intimal calcification is associated with late-stage atherosclerosis with a passive deposition of hydroxyapatite (calcium phosphate) into necrotic and apoptotic lesions of the plaques (11). In areas with tissue degeneration in the vascular wall, hydroxyapatite precipitation occurs when the physiological calcium phosphate concentration exceeds the threshold of solubility (12). On the other hand, medial calcification, such as that in Mönckeberg's medial sclerosis results from active processes with some similarities to osteogenesis (13). The vascular calcification process has been proposed (14). Inflammation triggered by elastin degradation, lipid deposition or pathogens in the vascular wall induces activation and proliferation. Activated macrophages induce proliferation and differentiation of neighboring VSMCs or stem cells with mesenchymal differentiation potential into osteogenic precursor cells. Through secreted molecules and direct cell-cell interaction, the osteogenic precursor cells differentiate to osteoblast-like cells. These osteoblast-like cells deposit bone matrix proteins that are subsequently mineralized. At that time, matrix vesicles and apoptotic bodies from VSMCs functioning as the nidus for mineral nucleation facilitate vascular calcification. In the process, the gain of calcification promoters, such as bone morphogenetic proteins (BMPs) and the loss of calcification inhibitors, such as matrix Gla protein (MGP) and fetuin-A (also known as $\alpha 2$-HeremansSchmid glycoprotein or $\alpha$-2-HS glycoprotein) are involved. The factors governing bone formation overlap with those of vascular calcification (15).

The development of proteomic analyses and cDNA microarray techniques has made it possible to examine comprehensive differential expression of numerous proteins or genes between two conditions. Recently, proteomic analyses have been applied to clarify molecular alterations in AAAs (16). cDNA microarray analysis has also been applied to diverse disease processes including AAAs (17) and TAAs (18). However, the spectrum of molecular alterations that may occur in calcified aneurysms remains unknown.

The purpose of this study was to establish proteomic profiles of differentially expressed proteins in calcified abdominal aortic aneurysms (CAAs) and calcified thoracic aortic aneurysms (CTAs) relative to adjacent normal aorta tissues. Recently, a variety of large-scale mass spectrometry (MS)-based analyses with high sensitivity for detection and quantification of differentially expressed proteins between two conditions have been developed. In order to uncover proteins that are significantly changed in CAAs and CTAs, the isobaric tag for absolute and relative quantitation (iTRAQ) technology (19) coupled with nano liquid chromatography (nanoLC)-matrix-assisted laser desorption ionization (MALDI)-time of flight (TOF/TOF)-tandem MS (MS/MS) followed by ProteinPilot analysis (20) was used in this study as a quantitative proteomic approach. We performed a detailed characterization of CAAs and CTAs and revealed differences between normal aorta tissues and CAAs or CTAs.

\section{Materials and methods}

Patients and samples. The participants gave informed consent, and the studies were performed with the approval of the Ethics Committee of Shimane University School of Medicine, Japan. Calcified aortic aneurysm tissues were obtained at surgery from subjects with CAAs ( $\mathrm{n}=6)$ and from subjects with CTAs $(n=6)$ who underwent elective aneurysm repair. Reference samples were obtained from normal aorta tissues adjacent to CAA or CTA tissues. Tissue samples were snap-frozen in liquid nitrogen and stored at $-80^{\circ} \mathrm{C}$ until protein extraction.

Sample preparation. Sample preparation was performed according to the manual supplied by AB Sciex (Foster, CA, USA). Briefly, $20 \mathrm{mg}$ of the CAA or CTA samples and neighboring normal aorta tissues as controls were subjected to protein extraction. After adding urea lysis buffer containing $7 \mathrm{M}$ urea, $0.1 \%$ Nonidet P-40 (NP-40) and $500 \mathrm{mM}$ triethylammonium bicarbonate (TEAB) (Sigma, Tokyo, Japan), the sample was sonicated 5 times for $10 \mathrm{sec}$ each time on ice using a Sonifier 450 sonicating probe (Branson Japan, Atsugi, Japan), incubated at $4^{\circ} \mathrm{C}$ for $1 \mathrm{~h}$ with constant shaking, and centrifuged at $10,000 \mathrm{x}$ g for $10 \mathrm{~min}$, and then the supernatant was collected. The supernatant was desalted and its buffer was exchanged with $50 \mathrm{mM}$ TEAB using spin concentrators (Corning, Tokyo, Japan). The protein concentration was determined using a bicinchoninic acid (BCA) assay (Pierce, Rockford, IL, USA).

Denaturation, reduction, trypsin digestion, and $i T R A Q$ labeling. Labeling with iTRAQ was performed using iTRAQ ${ }^{\mathrm{TM}}$ Reagent from AB Sciex as described in the manufacturer's instructions. Briefly, as described previously (20), each of $50 \mu \mathrm{g}$ of CAA or CTA samples and neighboring normal aorta samples was denatured by sodium dodecyl sulfate (SDS) and reduced by [tris-(2-carboxyethyl)phosphine (TCEP)] as described previously. Then cysteine alkylation was performed using methylmethanethiosulfonate (MMTS). Subsequently, each sample was cleaved by trypsin. Digests were labeled with an iTRAQ Reagent Multiplex kit (AB Sciex). iTRAQ label 114 or 115 was used for labeling the control sample, and iTRAQ label 116 or 117 was used for labeling the CAA or CTA sample. Then the labeled control and the calcified aneurysmal samples were combined. 
A

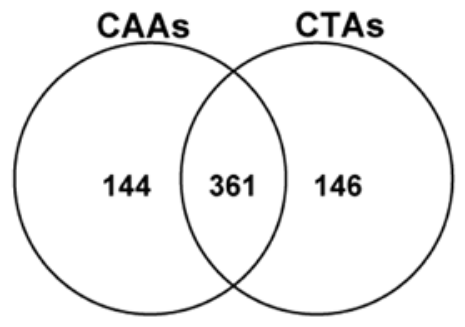

B

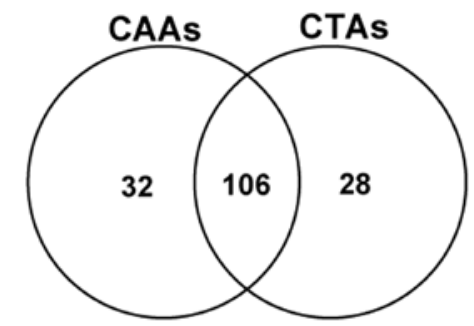

C

\begin{tabular}{|c|c|c|}
\hline \multirow{2}{*}{ iTRAQ ratio } & \multicolumn{2}{|c|}{ Number of proteins } \\
\cline { 2 - 3 } & CAAs & CTAs \\
\hline$\geqq 1.3$ & 41 & 28 \\
\hline$<0.77$ & 4 & 60 \\
\hline
\end{tabular}

Figure 1. Venn diagrams presenting the numbers of differentially expressed proteins of CAAs, CTAs, and both calcified aneurysms compared with adjacent normal aortic tissues. (A) Number of proteins that were identified in more than one sample among 6 samples with iTRAQ ratios. (B) Number of proteins that were identified in at least 5 samples among 6 samples with iTRAQ ratios. (C) Numbers of proteins with significantly increased ( $\geq 1.3$-fold) and decreased expression ratios $(<0.77$-fold) that were identified in at least 5 samples among 6 samples in CAAs and CTAs.

Strong cation exchange (SCX) chromatograpy and nanoLC. SCX chromatography was performed with the AB Sciex cation exchange system according to the manufacturer's instructions. Subsequently, 6 fractions were collected and desalted by a Sep-Pac $\mathrm{C}_{18}$ cartridge according to the instructions of Waters Corporation (Milford, MA, USA). Fractionation with a NanoLC system was performed according to the instructions of the manufacturer (KYA Technologies, Tokyo, Japan). A total of 171 fractions per one fraction of SCX chromatography were collected onto an Opti-TOF LC/MALDI 384 target plate (AB Sciex) using a DiNa Map fraction collector (KYA Technologies). The methods for this step have been described in detail in our previous paper (20).

$M S$ analysis and protein identification and relative quantification. The Mass Spectrometer 5800 MALDI-TOF/ TOF Analyzer (AB Sciex) with TOF/TOF Series software (version 4.0) was used for MS data acquisition in a positive ion mode. Monoisotopic precursor selection for MS/MS was performed by automatic precursor selection with an interpretation method using the DynamicExit Algorithm (AB Sciex). The peptide data obtained by 5800 MALDI TOF/TOF MS/ MS were analyzed with ProteinPilot ${ }^{\mathrm{TM}} 3.0$ software using the Paragon protein database search algorithm (AB Sciex) (21). Each MS/MS spectrum was searched against the database constructed by AB Sciex (version 20081216; 20,489 entries).
Bioinformatic analysis. The Panther system (version 7) (http://www.pantherdb.org/) was used for classification and pathway analyses of proteins. The annotations of proteins were obtained from the Uniprot database (http://www.uniprot.org/) and appropriate literature. Clustering analysis of differentially expressed proteins was performed by the Genesis software provided by the Genesis team of Institute for Genomics and Bioinformatics, Graz University of Technology (Graz, Austria) (http://genome.tugraz.at/) (22).

Western blot analysis. Twenty micrograms of cell lysates extracted with urea lysis buffer as described above was electrophoresed through 10\% SDS-polyacrylamide gel (PAGE), and proteins were subsequently electroblotted onto Hybond ECL nitrocellulose membranes (GE Healthcare Japan, Hino, Japan). The membranes were blocked at room temperature for $1 \mathrm{~h}$ with $5 \%$ nonfat dry milk in TBST $[20 \mathrm{mM}$ Tris- $\mathrm{HCl}$ (pH 7.5), $150 \mathrm{mM} \mathrm{NaCl}, 0.1 \%$ Tween-20] and then incubated with rabbit polyclonal anti- $\alpha-2-H S$-glycoprotein antibody $(0.5 \mu \mathrm{g} / \mathrm{ml}$ in TBST with $1 \%$ milk) (Calbiochem, Darmstadt, Germany), rabbit polyclonal anti-ceruloplasmin antibody (Epitomics, Burlingame, CA, USA), mouse monoclonal antiperiostin antibody (Sigma-Aldrich, St. Louis, MO, USA), or rabbit polyclonal anti-TGM2 antibody (Epitomics) for $1 \mathrm{~h}$ at room temperature. Membranes were washed three times with TBST and incubated with anti-rabbit or anti-mouse Alexa Fluor 680-conjugated or IRDye 800-conjugated immunoglobulin $(\mathrm{Ig}) \mathrm{G}$, followed by visualization using the infrared imaging system Odyssey (LI-COR, Inc., Lincoln, NE, USA). Data from duplicate experiments were analyzed for statistical significance by the unpaired t-test. Statistical significance was set at the $95 \%$ level $(\mathrm{P}<0.05)$.

\section{Results}

Quantitative analysis of differentially expressed proteins between CAA or CTA and adjacent normal aorta tissues. A total of 6 calcified abdominal aortic aneurysmal tissues and adjacent normal aorta tissues were collected from 6 CAA patients. Similarly, 6 calcified thoracic aortic aneurysmal tissues and adjacent normal aorta tissues were collected from 6 CTA patients. Protein expression was compared between each calcified aneurysmal tissue and adjacent normal artery using iTRAQ labeling coupled to nanoLC-MALDI-TOF/ TOF-MS/MS followed by ProteinPilot analysis. A total of 505 differentially expressed proteins in 6 CAA samples and 507 proteins in 6 CTA samples with 361 proteins in both calcified aneurysmal tissues that were identified in more than one sample were shown (Fig. 1A). Among them, 138 differentially expressed proteins in 6 CAA samples and 134 proteins in 6 CTA samples with 106 proteins in both calcified aneurysmal tissues that were identified in at least 5 samples were shown using highly stringent criteria for protein identification (unused ProtScore $>2$; confidence $>99 \%$ ) (Fig. 1B). The relative quantitation by ProteinPilot analysis was based on statistical analysis. However, since most biochemical methods are apt to include technical variation, we considered an additional cut-off value at 1.3 -fold change ( $\geq 1.3$ - or $<0.77$-fold) for iTRAQ ratios to reduce false positive rates for the selection of differentially expressed proteins. Finally, among them 41 and 


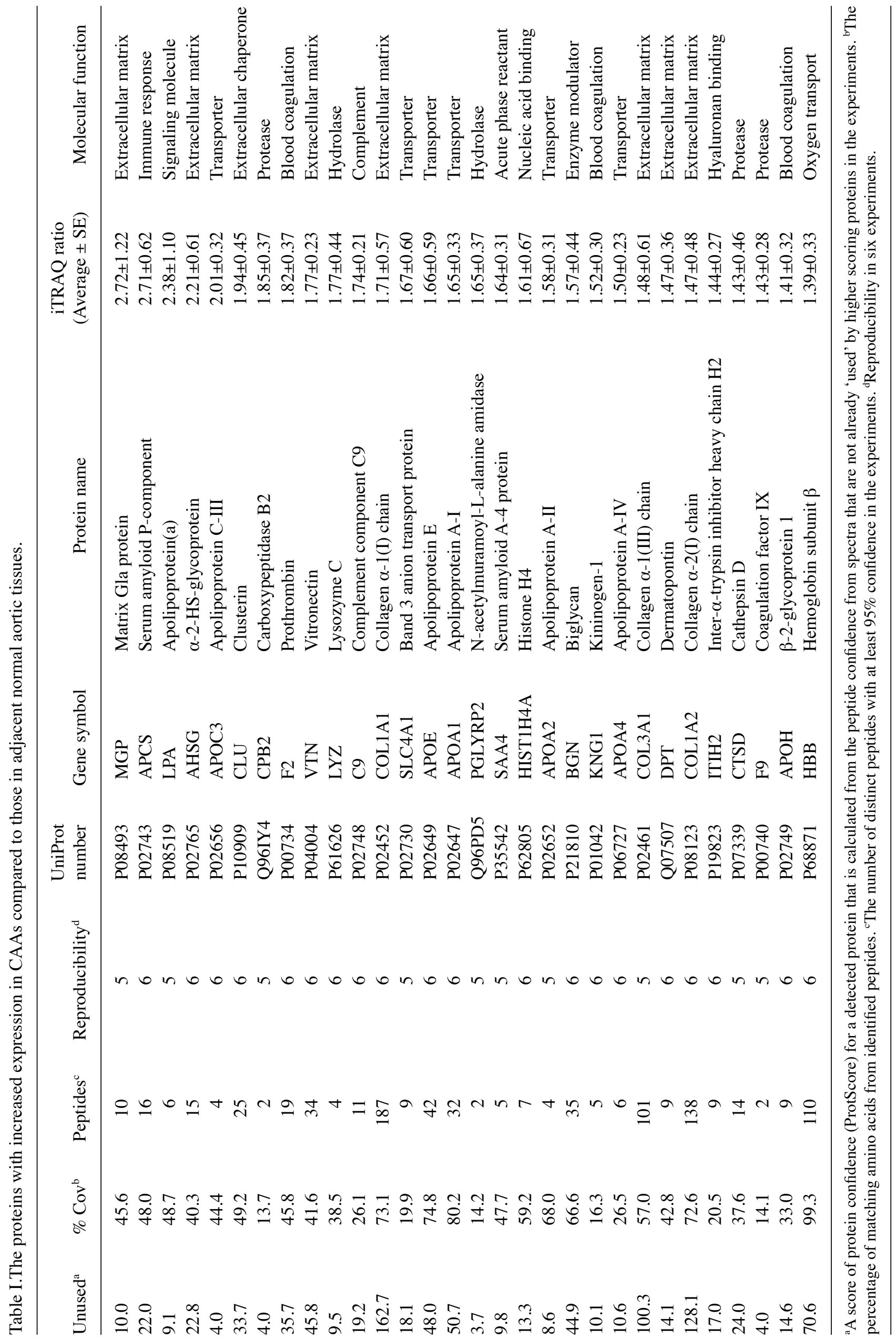


28 proteins with significantly increased expression ( $\geq 1.3$-fold) and 4 and 60 proteins with significantly decreased expression $(<0.77$-fold) were identified in CAAs and CTAs, respectively (Fig. 1C).

In Tables I-IV, increased and decreased proteins in CAAs and CTAs compared with those in adjacent normal aorta tissues are listed in the order of iTRAQ ratios. Among them, proteins such as type I and type III collagens $(16,23)$ in increased proteins in CAAs, type I collagen, fibrinogen $\alpha, \beta, \gamma$ chains and prothrombin (24) in increased proteins in CTAs, and fibulin-5 (DANCE) (25) in decreased proteins in CTAs that are known to be involved in aortic aneurysmal development and progression were identified. Proteins such as matrix Gla protein, $\alpha$-2-HS-glycoprotein (fetuin-A) (15) and biglycan in increased proteins in CAAs, mimecan (osteoglycin) in decreased proteins in CAAs, $\alpha-2-H S$-glycoprotein, and decorin and biglycan (26) in increased proteins in CTAs that are known to participate in vascular calcification were also identified.

Molecular function classification of differentially expressed proteins. For the functional distribution, analysis of the identified proteins with significant differential expression $(\geq 1.3$ - or $<0.77$-fold) in CAAs and CTAs was performed using the Panther classification system, which sorts the proteins into respective classes based on their biological process (Fig. 2). We classified the identified variable proteins with increased expression in the following groups with more than 4 proteins: transporter [7 proteins $(17.1 \%)$ ], signaling molecule [6 proteins $(14.6 \%)]$, protease [5 proteins $(12.2 \%)$ ] and transfer/carrier protein [4 proteins $(9.8 \%)]$ among 41 proteins of CAAs, and signaling molecule [8 proteins $(28.6 \%)$ ] and enzyme modulator [4 proteins (14.3\%)] among 28 proteins of CTAs. On the other hand, the groups with decreased expression were cytoskeletal protein [13 proteins $(21.7 \%)$ ], signaling molecule [8 proteins $(13.3 \%)]$, transfer/carrier protein [5 proteins $(8.3 \%)]$, isomerase [4 proteins $(6.7 \%)]$, oxidoreductase [4 proteins $(6.7 \%)]$, and structural proteins [4 proteins $(6.7 \%)]$ among 60 proteins of CTAs. There were no classified groups for decreased proteins in CAAs. The transporter group included apolipoprotein C-III (apoC-III), band 3 anion transport protein, apolipoprotein $\mathrm{E}$ (apoE), apolipoprotein A-I (apoA-I), serum amyloid A-4 protein, apolipoprotein A-II (apoA-II), and apolipoprotein A-IV (apoA-IV) with increased expression in CAAs. The signaling molecule group included fibrinogen $\gamma, \beta, \alpha$ chains, periostin, thrombospondin-1, complement Clq subcomponent subunit $\mathrm{C}$, complement $\mathrm{C} 3$, and type I collagen $\alpha-1$ with increased expression in CTAs. The cytoskeletal protein group included transgelin, myosin regulatory light polypeptide 9 , tropomyosin $\beta$ chain, cysteine and glycine-rich protein 1, calponin-1, caldesmon, lipoma-preferred partner, myosin light polypeptide 6 , talin-1, moesin, vinculin, PDZ and LIM domain protein 7 , and gelsolin with decreased expression in CTAs.

Pathway analysis of differentially expressed proteins. The Panther software was also used for pathway analyses of proteins that were shown to have significant differential expression ( $\geq 1.3-$ or $<0.77$-fold) in our study. It was notable that 6 proteins with increased expression in both CAAs and CTAs correspond to proteins found in the blood coagulation 


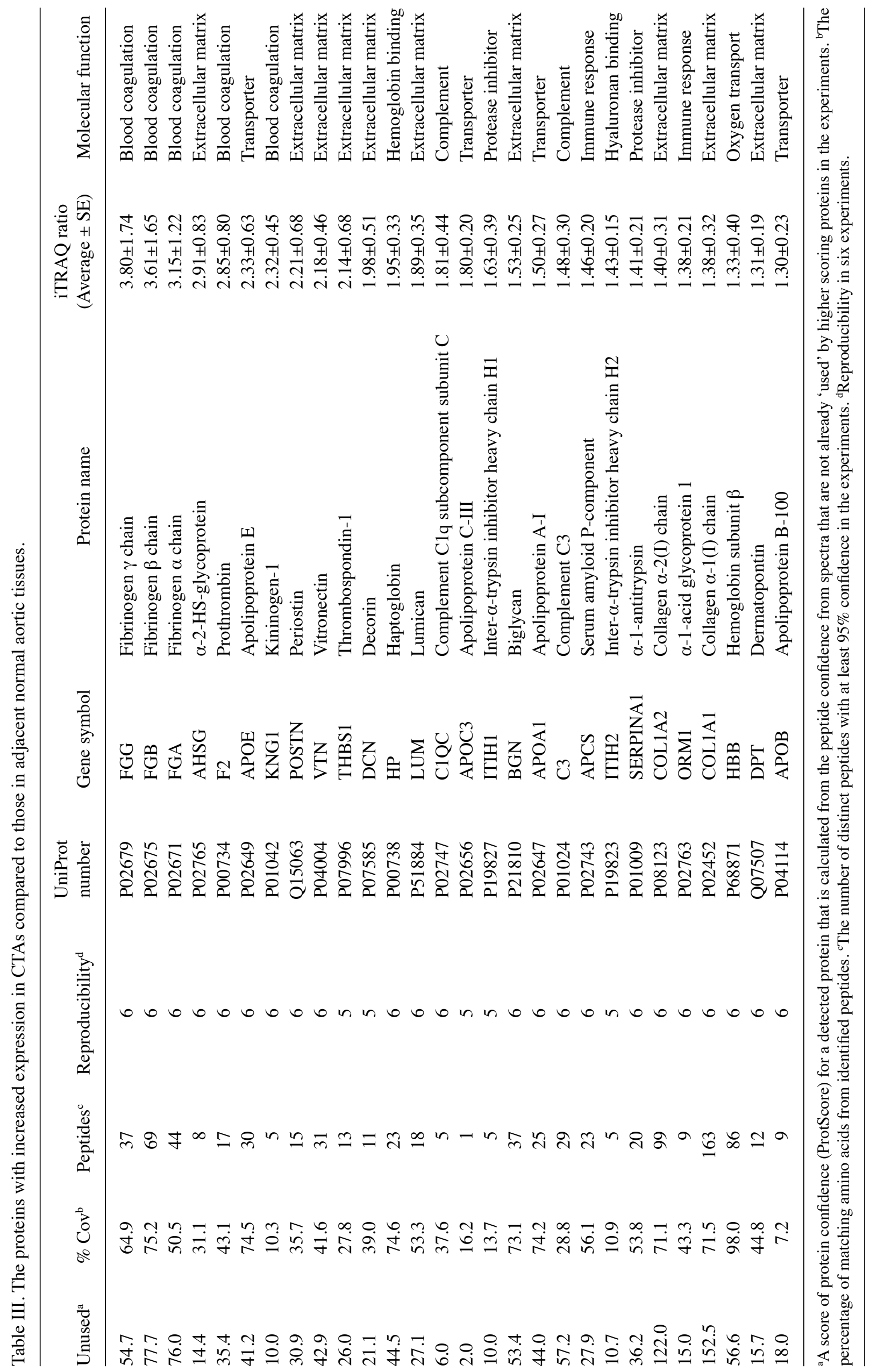


Number of classified decreased proteins

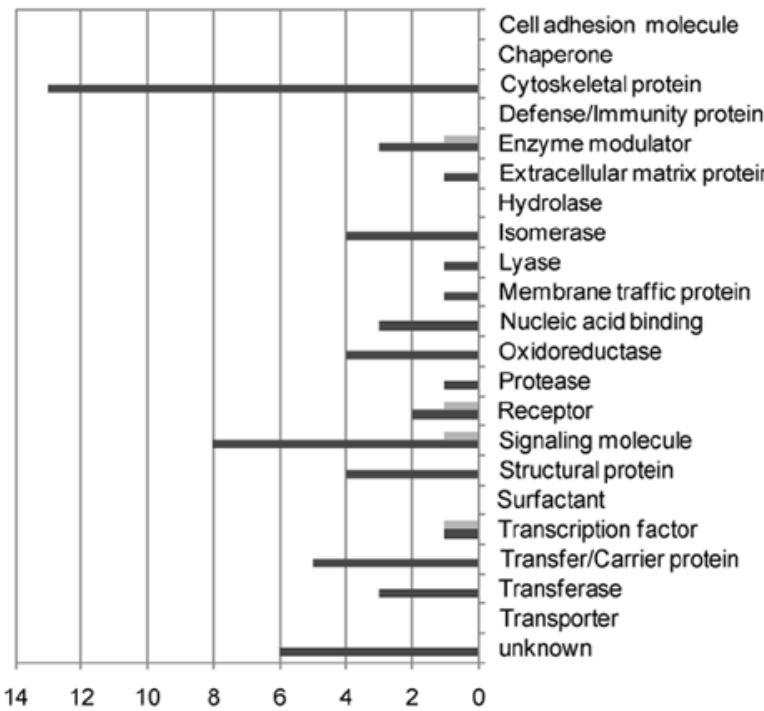

Number of classified increased proteins

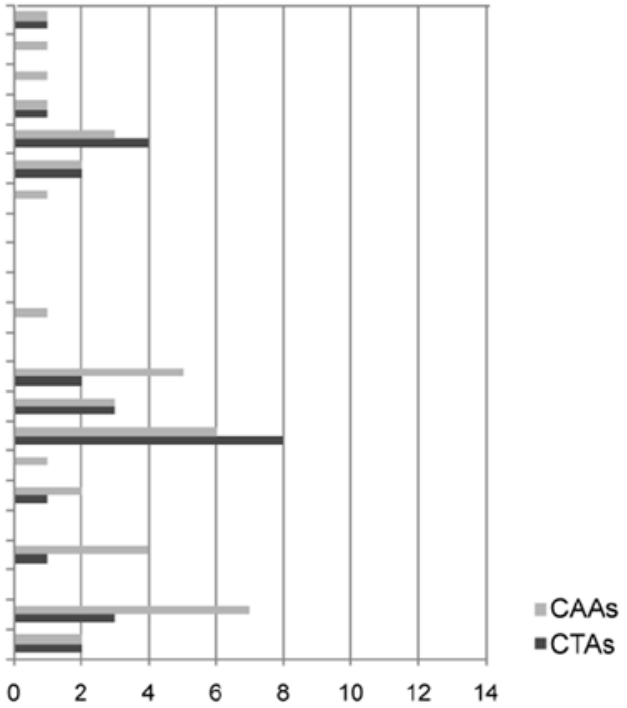

Figure 2. Classification analysis of proteins that show significantly increased ( $\geq 1.3$-fold) and decreased expression $(<0.77$-fold) in CAAs and CTAs by the Panther software. Number of increased or decreased proteins are shown in each group.

Number of decreased proteins

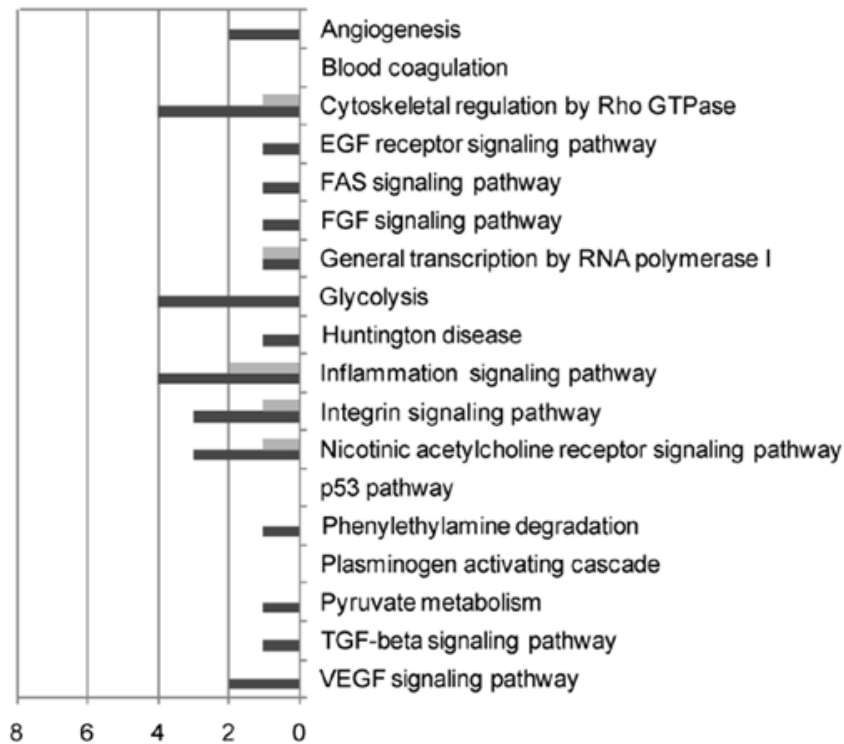

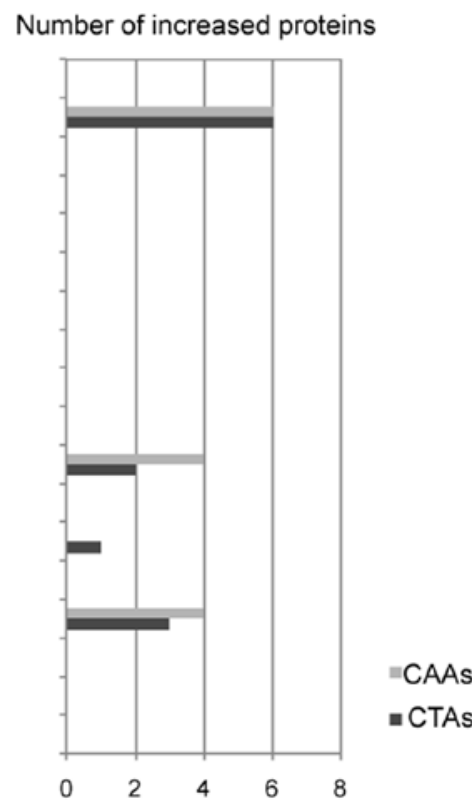

Figure 3. Pathway analysis of proteins that show significantly increased ( $\geq 1.3$-fold) and decreased expression ( $<0.77$-fold) in CAAs and CTAs by the Panther software. Number of increased or decreased proteins are shown in each pathway.

pathway illustrated in Fig. 3. In addition, two pathways each consisting of 4 proteins with increased expression in CAAs corresponded to proteins in the plasminogen activating cascade and the integrin signaling pathway. On the other hand, three pathways consisting of each of 4 proteins with decreased expression in CTAs corresponded to proteins in the cytoskeletal regulation by Rho GTPase pathway, the glycolysis pathway, and the inflammation mediated by chemokine and cytokine signaling pathway.

Hierarchical clustering analysis of differentially expressed proteins in CAAs and CTAs. Cluster analysis with the Genesis software was performed for our identified proteins to evaluate the relation between differentially expressed proteins that were identified in at least 5 samples among 6 CAA samples or 6 CTA samples and to identify interesting protein expression clusters (Fig. 4A). We examined 138 differentially expressed proteins in CAAs and the 134 differentially expressed proteins in CTAs. Interestingly, in CAAs, three proteins with increased expression (type I collagen $\alpha-1$ and $\alpha-2$, and type III collagen $\alpha-1$ ) included in the integrin signaling pathway (Fig. 3) were clustered (Fig. 4A, left panel). On the other hand, in CTAs, three proteins with increased expression (fibrinogen $\alpha, \beta, \gamma$ chains) included in the blood coagulation pathway (Fig. 3), three proteins with decrease expression (myosin-10, -11 and type XIV collagen $\alpha-1$ ) included in the 
A

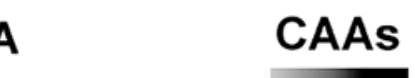

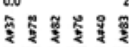
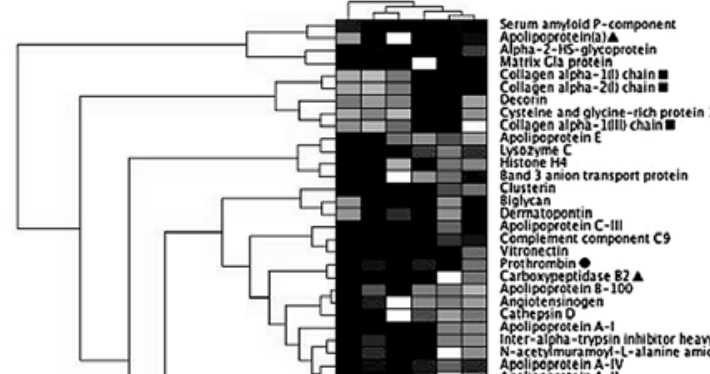

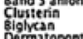

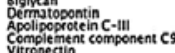

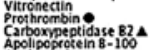

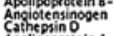
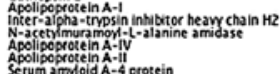

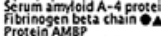

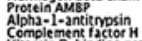

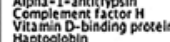

Vitainin D-bi:
kaptoglobin
Hemoolexin

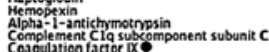

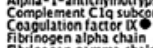

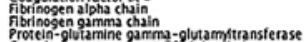

Complement component c8alpha thain

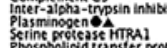

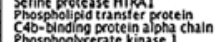

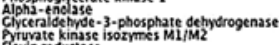

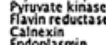

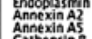

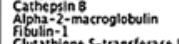

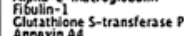

chatsise

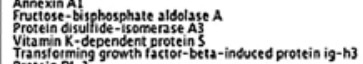

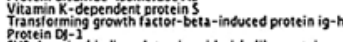

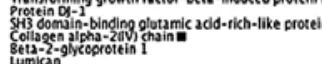

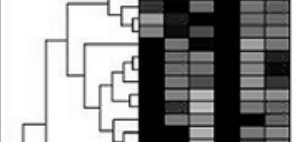

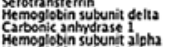

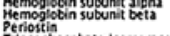

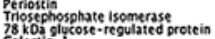

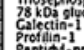

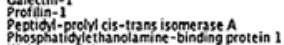

and

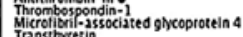

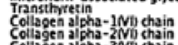

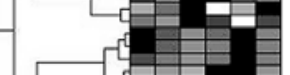

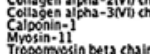

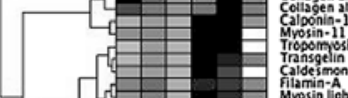

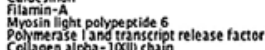

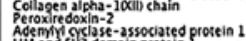

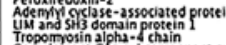
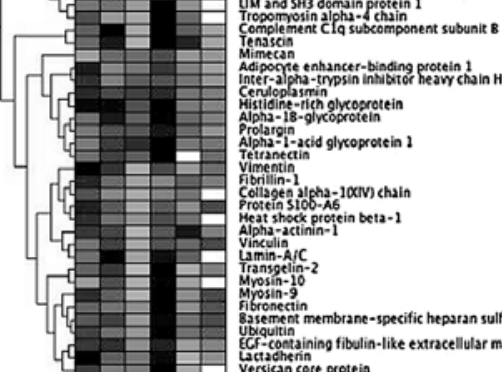

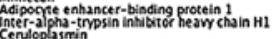

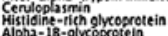

Prolargin-acid glycoprotein

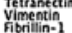

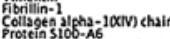

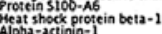

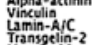

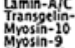

Gibronectin

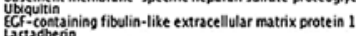

Ecr-containing fibulin-
Letarthenin
versican core protein

\section{CTAs}

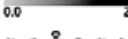

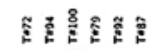

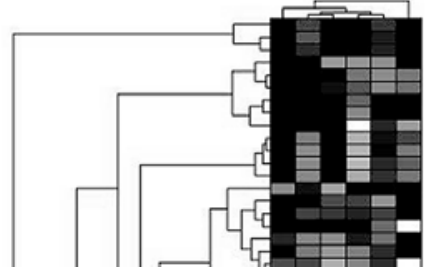

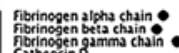

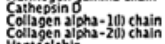

capteglobin

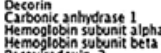

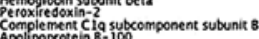

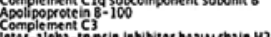

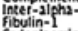

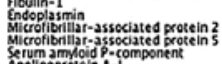

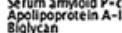

Bagram

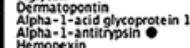

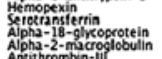

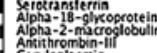

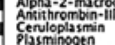

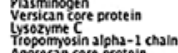

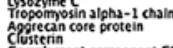

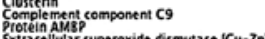

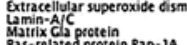

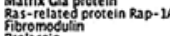

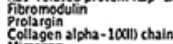

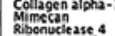

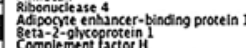

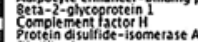

Protein Gistufice-isometase A3

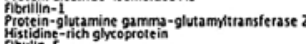

Histidine-tich
Bbollin-5
Calponin-1

Calonin-1
clocimon
tropomposin beta chain
cysteline and glycine-rich protein 1

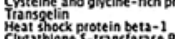

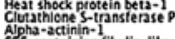

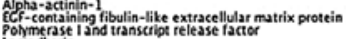

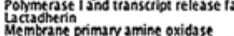

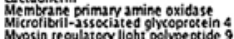

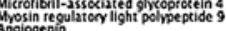

ont
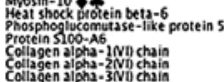

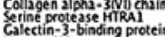

Histone H4

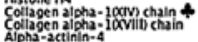

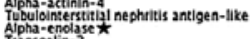

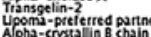

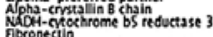

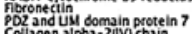

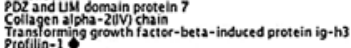

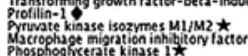

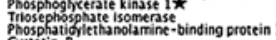

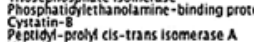

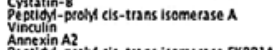

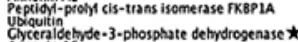

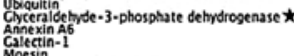

Moesin light porpeptide 6
Virmentig

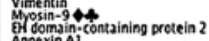

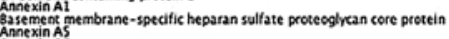

AmexnAs As

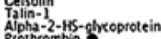

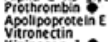

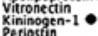

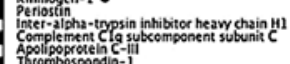

Figure 4A. Hierarchical clustering analysis of differentially expressed proteins in CAAs and CTAs by Genesis software. (A) The dendrogram displays two-way hierarchical clustering analyses of 138 differentially expressed proteins that were commonly identified in at least 5 samples among 6 CAA samples (A\#37, A\#40, A\#76, A\#78, A\#82 and A\#83) (left panel) and 134 differentially expressed proteins that were identified in at least 5 samples among 6 CTA samples (T\#72, T\#79, T\#87, T\#92, T\#94 and T\#100) (right panel). Differences in iTRAQ ratios are color-coded. Darker black spectra indicate increased expression, whereas lighter grey spectra indicate decreased expression compared with those in adjacent normal aorta tissues. White squares show no detection of indicated proteins in corresponding samples. Type I collagen $\alpha-1$ and $\alpha-2$ chains, type III collagen $\alpha-1$ chain and type IV collagen $\alpha-2$ chain included in the integrin signaling pathway with increased expression in CAAs (Fig. 3) are indicated with black squares in the left panel. Prothrombin, fibrinogen $\beta$ chain, coagulation factor IX, plasminogen, kininogen-1 and antithrombin-III included in the blood coagulation pathway with increased expression in CAAs (Fig. 3) are indicated with black circles in the left panel. Apolipoprotein(a), carboxypeptidase B2, fibrinogen $\beta$ chain and plasminogen included in the plasminogen activated cascade with increased expression in CAAs (Fig. 3) are indicated with black triangles in the left panel. Fibrinogen $\alpha, \beta$ and $\gamma$ chains, $\alpha$-1-antitrypsin, prothrombin and kininogen-1 included in the blood coagulation pathway with increased expression in CTAs (Fig. 3) are indicated with black circles in the right panel. Myosin-11, myosin-10, profilin-1 and myosin-9 included in the cytoskeletal regulation by Rho GTPase pathway with decreased expression in CTAs (Fig. 3) are indicated with black diamonds in the right panel. Myosin-11, myosin-10, type XIV collagen $\alpha-1$ and myosin- 9 included in the inflammation mediated by chemokine and cytokine signaling pathway with decreased expression in CTAs (Fig. 3) are indicated with black clover-shapes in the right panel. $\alpha$-enolase, pyruvate kinase isozymes M1/M2, glyceraldehyde-3-phosphate dehydrogenase and phosphoglycerate kinase 1 included in the glycolysis pathway with decreased expression in CTAs (Fig. 3) are indicated with black stars in the right panel. 
B

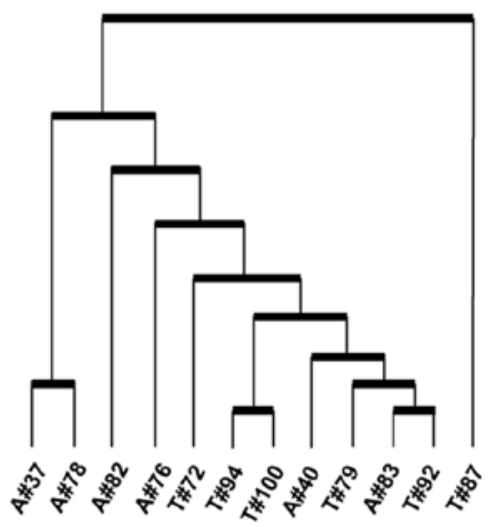

Figure 4B. Hierarchical clustering analysis of differentially expressed proteins in CAAs and CTAs by Genesis software. (B) Hierarchical clustering of 12 calcified aortic aneurysmal samples with 6 CAAs and 6 CTAs based on differentially expressed proteins compared with those in adjacent normal aorta tissues. One hundred forty differentially expressed proteins that were commonly identified in at least 9 samples among 12 samples (A\#37, A\#40, A\#76, A\#78, A\#82, A\#83, T\#72, T\#79, T\#87, T\#92, T\#94 and T\#100) were analyzed.

inflammation mediated by chemokine and cytokine signaling pathway (Fig. 3), and three proteins with decreased expression (pyruvate kinase isozymes M1/M2, phosphoglycerate kinase 1, and glyceraldehyde-3-phosphate dehydrogenase (GAPDH)) included in the glycolysis pathway (Fig. 3) were clustered (Fig. 4A, right panel).

Subsequently, we performed cluster analysis of 12 calcified aortic aneurysmal samples from 6 CAA and 6 CTA patients based on the expression patterns of differentially expressed proteins of each sample (Fig. 4B). Samples of CAAs (patient nos. A\#37, A\#76, A\#78 and A\#82) and samples of CTAs (T\#72, T\#79, T\#87, T\#92, T\#94 and T\#100) were properly clustered. Interestingly, CAA samples from patients with both CAAs and CTAs (A\#40 and A\#83) were clustered outside the samples of patients with CAAs and were rather clustered with samples of patients with CTAs.

Validation of $i T R A Q$ ratio by western blot analysis. To validate the accuracy of the quantitative results by iTRAQ ratios of differentially expressed proteins, some proteins with an iTRAQ quantitative ratio were quantified again by western blot analysis. Ceruloplasmin (iTRAQ ratio, 1.00) in CAAs and protein-glutamine $\gamma$-glutamyltransferase (referred to as TGM2) (iTRAQ ratio, 0.99) in CTAs were used as proteins expressed equivalently in a calcified aortic aneurysm and normal adjacent blood vessel. On the other hand, $\alpha-2-H S$-glycoprotein (referred to as AHSG) (iTRAQ ratio, 2.21) in CAAs and periostin (iTRAQ ratio, 2.21) in CTAs were selected as representative proteins, since the iTRAQ ratios of these proteins were relatively large. We performed western blot analysis with anti-AHSG antibody and anti-ceruloplasmin antibody against CAA and adjacent normal aortic samples from patient A\#40 (Fig. 5Aa). The ratio of increased expression of AHSG in the CAA normalized with ceruloplasmin expression was determined by band intensity and it was 2.22 -fold (Fig. 5Ab). This value is consistent with the quantitative result determined by the iTRAQ ratio (2.21). Similarly, western blot analysis was performed with anti-periostin antibody and anti-TGM2 antibody against CTA and adjacent normal aortic samples from
A

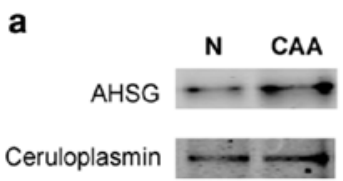

b

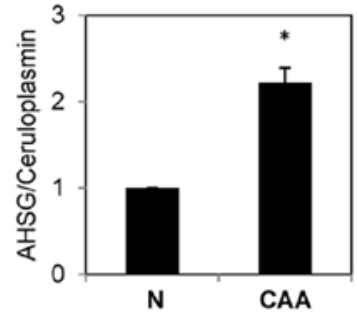

B

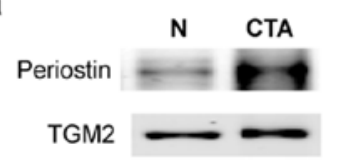

b

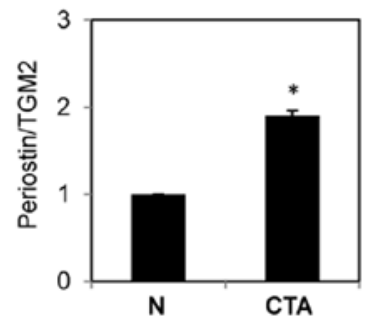

Figure 5. Validation of iTRAQ ratio by western blotting. (Aa) Western blot analysis with anti- $\alpha$-2-HS-glycoprotein (referred to as AHSG) antibody and anti-ceruloplasmin antibody against CAA and adjacent normal aorta $(\mathrm{N})$ samples. (Ab) Densitometric analysis in duplicated experiments for AHSG. The ratio of increased expression (2.22-fold) of AHSG normalized with ceruloplasmin expression in CAA compared with those of adjacent normal aorta samples was determined by band intensity. (Ba) Western blot analysis with anti-periostin antibody and anti-protein-glutamine $\gamma$-glutamyltransferase (TGM2) antibody against CTA and adjacent normal aorta (N) samples. (Bb) Densitometric analysis in duplicated experiments for periostin. The ratio of increased expression (1.90-fold) of periostin normalized with TGM2 expression in CTA compared with those of adjacent normal aorta samples was determined by band intensity. ${ }^{*} \mathrm{P}<0.05$.

patient T\#94 (Fig. 5Ba). The ratio of increased expression of periostin in the CTA normalized with TGM2 expression was 1.90 -fold (Fig. 5Bb). This value is nearly consistent with the quantitative result determined by the iTRAQ ratio (2.21). Overall, there was a good correlation between the iTRAQ ratio and band intensity of western blot analysis.

\section{Discussion}

Calcified aortic aneurysm is a cardiovascular disease. It is a complex disease with both genetic and various risk factors, including aging, gender, and inflammation factors, contributing to the development and progression of the disease. The molecular pathogenesis of calcified aortic aneurysm is poorly understood. In this study, we carried out a global proteomic study on calcified aneurysmal tissues from abdominal and thoracic aortas and adjacent normal aorta tissues. Quantitative proteomics using iTRAQ labeling followed by nanoLC-MALDI-TOF/ TOF-MS/MS analysis, which is currently one of the most widely used approaches for high-throughput protein quantitation, has been proven to be a useful technique for examination of molecular mechanisms in diseases. We used the iTRAQ proteomic approach to identify proteins with differential expression between CAAs or CTAs and their adjacent normal aorta tissues. As a result, 41 and 28 proteins with increased expression ( $\geq 1.3$-fold) and 4 and 60 proteins with decreased 


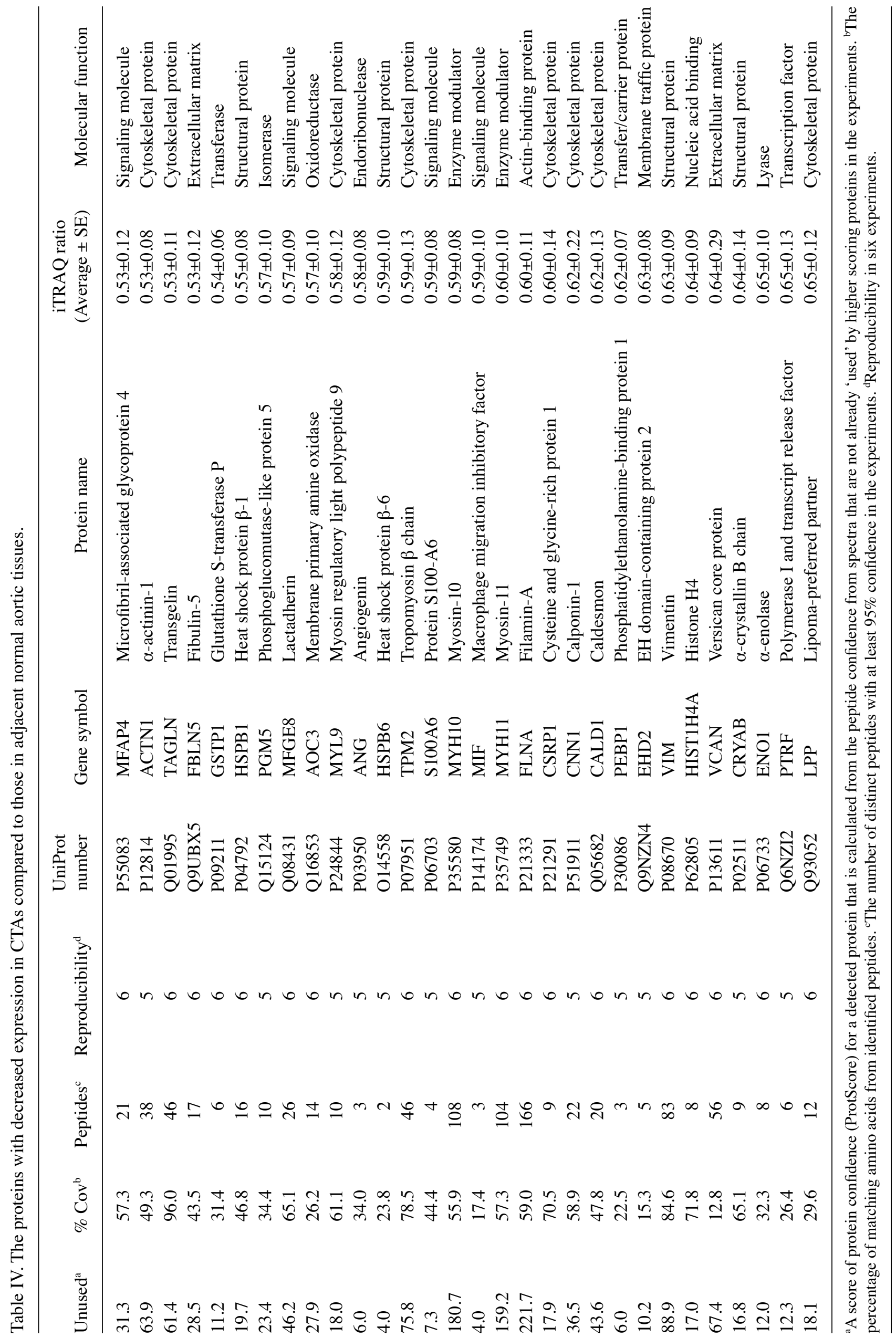


expression $(<0.77$-fold) were shown to be proteins with significant differential expression in CAAs and CTAs versus adjacent normal aorta tissues, respectively.

Among the proteins with significant differential expression in CAAs, type I collagen $\alpha-1$ and type III collagen $\alpha-1$ chains were increased, whereas type XIV collagen $\alpha-1$ was decreased. Collagen plays an important role in arterial wall integrity (27). Appropriate amounts of these collagens are essential for arterial integrity to endure the forces exerted by blood pressure. Either increased or decreased collagen in aortas may weaken the integrity of the aortic wall, resulting in predisposition of aortic aneurysms. Interestingly, increased amounts of collagens would lead to fibrosis, which may result in arterial stiffness and increased susceptibility to aneurysmal diseases. Similarly, not only collagens, but also other extracellular matrix proteins such as fibulin-5 (also known as DANCE) with regulated expression is essential for the functional integrity of vessel walls. Fibulin-5 showed significantly decreased expression in CTAs in this study. Fibulin-5 is essential for elastogenesis with an elastogenic organizer activity (28).

Vascular calcification is frequently detected in advanced atherosclerosis and aneurysms. Vascular calcification is a highly regulated process with inducers and inhibitors responsible for calcium deposition. An imbalance of these regulators results in the initiation of calcification. Under normal conditions, inhibitor proteins such as matrix Gla protein, BMP-7, $\alpha$-2-HS glycoprotein (fetuin-A), osteoprotegerin and osteopontin prevent vascular calcification processes (29). Among these representative inhibitors, matrix Gla protein and $\alpha-2-\mathrm{HS}$ glycoprotein were increased in CAAs and CTAs in this study. These findings are consistent with previous observations by Spronk et al (30). The upregulation of these inhibitors in calcified aneurysms might be explained by assuming a feedback mechanism of upregulated production of these inhibitors, leading to the prevention of further calcium phosphate deposition.

The abdominal and thoracic aortas share similarities in their physical structures and cellular components. In this study, 16 proteins including serum amyloid P-component, $\alpha$-2-HS glycoprotein, apolipoprotein C-III, prothrombin, and vitronectin were significantly increased in both CAAs and CTAs ( $\geq 1.3$-fold) and 3 proteins including type XIV collagen $\alpha-1$ chain, myosin- 10 , and polymerase I and transcript release factor were significantly decreased in both CAAs and CTAs ( $<0.77$-fold). These proteins might provide the basis for the similar pathological phenotypes between CAAs and CTAs. However, heterogeneity also exists between abdominal and thoracic aortas. This difference contributes to vessel mechanics, proteolytic activity, signaling pathways, and atherosclerotic plaque deposition, leading to disparities in the pathologic mechanisms involved in CAAs and CTAs. In the present study, 3 proteins (histone H4, serine protease HTRA1, and cysteine and glycine-rich protein 1) that were significantly increased in CAAs but significantly decreased in CTAs were identified. On the other hand, 10 proteins (including matrix Gla protein, clusterin, lysozyme $\mathrm{C}$, complement component $\mathrm{C}$, and cathepsin D) that were significantly increased only in CAAs, one protein (mimecan) that was significantly decreased only in CAAs, 12 proteins (including fibrinogen $\gamma$ and $\alpha$ chains, periostin, thrombospondin-1, and haptoglobin) that were significantly increased only in CTAs, 35 proteins (including microfibril-associated glycoprotein 4, $\alpha$-actinin-1, transgelin, glutathione S-transferase $\mathrm{P}$, and heat-shock protein $\beta-1)$ that were significantly decreased only in CTAs were identified. These proteins with distinct expression patterns might be involved in the different pathogenesis associated with these two types of calcified aneurysms.

Although it is still controversial as to whether atherosclerotic lesions are a prerequisite for the formation of AAAs, it was found with the Panther classification system that several kinds of apolipoproteins such as apoC-III, apoE, apoA-I, apoA-II, and apoA-IV exhibited increased expression in CAAs compared with their expression in control tissues in this study. Apolipoproteins are deeply involved in lipid metabolism. The development of atherosclerosis is associated with disorders of lipoprotein metabolism (31). Golledge et al (32) demonstrated that a low high-density lipoprotein level is the most sensitive predictor of AAA among lipid markers. However, several lines of evidence have suggested potent anti-atherogenic roles of apoE (33), apoA-I (34), apoA-II (35), and apoA-IV (36) in contrast to the atherogenic role of apoC-III (37). In addition, Sadek et al (38) demonstrated the upregulation of ApoE mRNA synthesis in AAAs by microarray analysis. The upregulation of apolipoproteins with anti-atherogenic function in this study might be explained by assuming a feedback mechanism of increased anti-atherogenic apolipoproteins, possibly to prevent further atherosclerosis required for calcified aortic aneurysms.

The presence of a chronic intraluminal thrombus (ILT) is often found in aortic aneurysms. An ILT participates in aneurysmal dilatation. An ILT is a highly biologically active site in which fibrin formation following platelet and thrombin activation occurs. The ILT is a laminated structure with several layers of fibrin clots and an underlying hematic luminal layer with undegraded crosslinked fibrin (39). Consistent with these observations, the pathway analysis using Panther software in this study revealed that 6 proteins with increased expression in both CAAs and CTAs are involved in the blood coagulation pathway. Interestingly, Stec et al (40) showed strong linear relationships between plasma fibrinogen and cardiovascular diseases.

Several studies have suggested that oxidative stress is increased in aortic aneurysms and that modulation of reactive oxygen species (ROS) influences the formation of aortic aneurysms (41). In aortic aneurysms, ROS are generated by many infiltrating leukocytes, and generation of ROS leads to increased production of MMPs (42). Thus, it is important for protection of aortic aneurysms to reduce the activity of ROS-generating systems and/or to increase the levels of cellular antioxidants and antioxidant enzymes, such as superoxide dismutase (SOD) and glutathione S-transferase (GST). It has been reported that SOD activity was reduced in AAA tissue samples (43). In addition, it is known that GST is involved in the removal of products of potential atherogenic substances, such as lipid oxides (44). In line with this evidence, it was found in the present study that SOD (iTRAQ ratio, 0.77) and GST (iTRAQ ratio, 0.54) were significantly decreased in CTAs compared with those in normal tissues.

Increasing evidence suggests that oxidative stress has effects on gene expression, protein stability, and enzymatic 
activity. It has been reported that GAPDH is one of the targets for oxidative stress. GAPDH plays an important role in energy metabolism in the glycolytic pathway. Sukhanov et al (45) showed that proatherogenic molecule oxidized low-density lipoprotein (OxLDL) downregulates GAPDH by decreasing protein stability, resulting in a reduction in ATP levels. In the present study, it was found that GAPDH levels decreased in CTAs compared with that in controls. Although we have not shown whether oxidative stress is actually increased in aortic aneurysms in the samples used in this study, it is possible that the decreased level of GAPDH is due to the increase in oxidative stress in CTAs. Furthermore, other decreased glycolytic enzymes such as $\alpha$-enolase, pyruvate kinase isozymes M1/M2, and phosphoglycerate kinase 1 in the present study may also be targets for oxidative stress.

Interestingly, as shown in Fig. 4B, it was found that the expression patterns of differentially expressed proteins in CAAs from patients with both CAAs and CTAs (A\#40 and A\#83) show more resemblance to those in CTAs from patients with only CTAs than those in CAAs from patients with only CAAs. We speculated that the factors that influence the protein expression in CAAs may be diffusely secreted from CTAs of patients $\mathrm{A} \# 40$ and $\mathrm{A} \# 83$ and, in consequence, the protein expression patterns in CAAs from these patients might be more closely related to those in CTAs than those in CAAs.

In conclusion, this is the first comprehensive study on the mechanisms of development and progression of calcified abdominal aortic aneurysms (CAAs) and thoracic aortic aneurysms (CTAs) using an iTRAQ proteomics approach. This study revealed many novel proteins that had not been known to be associated with the abdominal and thoracic aortic aneurysm formation and vascular calcification.

\section{Acknowledgements}

We thank Yasuko Sonoyama for sample collection. This study was supported in part by Grants-in-Aid for Scientific Research from the Ministry of Education, Culture, Sports, Science and Technology of Japan.

\section{References}

1. Annambhotla S, Bourgeois S, Wang X, Lin PH, Yao Q and Chen C: Recent advances in molecular mechanisms of abdominal aortic aneurysm formation. World J Surg 32: 976-986, 2008.

2. Galis ZS and Khatri JJ: Matrix metalloproteinases in vascular remodeling and atherogenesis: the good, the bad, and the ugly. Circ Res 90: 251-262, 2002.

3. Kuivaniemi H, Platsoucas CD and Tilson III MD: Aortic aneurysms: an immune disease with a strong genetic component Circulation 117: 242-252, 2008.

4. Abdul-Hussien H, Soekhoe RG, Weber E, et al: Collagen degradation in the abdominal aneurysm: a conspiracy of matrix metalloproteinase and cysteine collagenases. Am J Pathol 170: 809-817, 2007

5. Henderson EL, Geng YJ, Sukhova GK, Whittemore AD, Knox J and Libby P: Death of smooth muscle cells and expression of mediators of apoptosis by $\mathrm{T}$ lymphocytes in human abdominal aortic aneurysms. Circulation 99: 96-104, 1999.

6. Golledge J, Muller J, Daugherty A and Norman P: Abdominal aortic aneurysm: pathogenesis and implications for management. Arterioscler Thromb Vasc Biol 26: 2605-2613, 2006.

7. Reed D, Reed C, Stemmermann G and Hayashi T: Are aortic aneurysms caused by atherosclerosis? Circulation 85: 205-211, 1992.
8. Ruddy JM, Jones JA, Spinale FG and Ikonomidis JS: Regional heterogeneity within the aorta: relevance to aneurysm disease. $\mathrm{J}$ Thorac Cardiovasc Surg 136: 1123-1130, 2008.

9. Lesauskaite V, Tanganelli P, Sassi C, et al: Smooth muscle cells of the media in the dilatative pathology of ascending thoracic aorta: morphology, immunoreactivity for osteopontin, matrix metalloproteinases, and their inhibitors. Hum Pathol 32: 1003-1011, 2001.

10. Coady MA, Rizzo JA, Goldstein LJ and Elefteriades JA: Natural history, pathogenesis, and etiology of thoracic aortic aneurysms and dissections. Cardiol Clin 17: 615-635, 1999.

11. Rumberger JA, Simons DB, Fitzpatrick LA, Sheedy PF and Schwartz RS: Coronary artery calcium area by electron-beam computed tomography and coronary atherosclerotic plaque area. A histopathologic correlative study. Circulation 92: 2157-2162, 1995.

12. Shanahan CM: Mechanisms of vascular calcification in renal disease. Clin Nephrol 63: 146-157, 2005.

13. Lehto S, Niskanen L, Suhonen M, Rönnemaa T and Laakso M: Medial artery calcification. A neglected harbinger of cardiovascular complications in non-insulin-dependent diabetes mellitus. Arterioscler Thromb Vasc Biol 16: 978-983, 1996.

14. Rusanescu G, Weissleder R and Aikawa E: Notch signaling in cardiovascular disease and calcification. Curr Cardiol Rev 4: 148-156, 2008.

15. Kendrick J and Chonchol M: The role of phosphorus in the development and progression of vascular calcification. Am J Kidney Dis 58: 826-834, 2011.

16. Didangelos A, Yin X, Mandal K, et al: Extracellular matrix composition and remodeling in human abdominal aortic aneurysms: a proteomics approach. Mol Cell Proteomics 10: M111.008128,2011.

17. Tung WS, Lee JK and Thompson RW: Simultaneous analysis of 1176 gene products in normal human aorta and abdominal aortic aneurysms using a membrane-based complementary DNA expression array. J Vasc Surg 34: 143-150, 2001.

18. Absi TS, Sundt III TM, Tung WS, et al: Altered patterns of gene expression distinguishing ascending aortic aneurysms from abdominal aortic aneurysms: complementary DNA expression profiling in the molecular characterization of aortic disease. J Thorac Cardiovasc Surg 126: 344-357, 2003.

19. Ross PL, Huang YN, Marchese JN, et al: Multiplexed protein quantitation in Saccharomyces cerevisiae using amine-reactive isobaric tagging reagents. Mol Cell Proteomics 3: 1154-1169, 2004.

20. Matsumoto K: Phosphorylation of extracellular matrix tenascin-X detected by differential mass tagging followed by nanoLC-MALDITOF/TOF-MS/MS using ProteinPilot software. Connect Tissue Res 53: 106-116, 2012.

21. Shilov IV, Seymour SL, Patel AA, et al: The Paragon Algorithm, a next generation search engine that uses sequence temperature values and feature probabilities to identify peptides from tandem mass spectra. Mol Cell Proteomics 6: 1638-1655, 2007.

22. Sturn A, Quackenbush J and Trajanoski Z: Genesis: cluster analysis of microarray data. Bioinformatics 18: 207-208, 2002.

23. Wang X, LeMaire SA, Chen L, et al: Increased collagen deposition and elevated expression of connective tissue growth factor in human thoracic aortic dissection. Circulation 114 (Suppl 1): I200-I205, 2006.

24. Touat Z, Lepage L, Ollivier V, et al: Dilation-dependent activation of platelets and prothrombin in human thoracic ascending aortic aneurysm. Arterioscler Thromb Vasc Biol 28: 940-946, 2008.

25. Wang X, LeMaire SA, Chen L, et al: Decreased expression of fibulin-5 correlates with reduced elastin in thoracic aortic dissection. Surgery 138: 352-359, 2005.

26. Wallin R, Wajih N, Greenwood GT and Sane DC: Arterial calcification: a review of mechanisms, animal models, and the prospects for therapy. Med Res Rev 21: 274-301, 2001.

27. Jensen LT and Høst NB: Collagen: scaffold for repair or execution. Cardiovasc Res 33: 535-539, 1997.

28. Nakamura T, Lozano PR, Ikeda Y, et al: Fibulin-5/DANCE is essential for elastogenesis in vivo. Nature 415: 171-175, 2002.

29. Rezg R, Barreto FC, Barreto DV, Liabeuf S, Drüeke TB and Massy ZA: Inhibitors of vascular calcification as potential therapeutic targets. J Nephrol 24: 416-427, 2011.

30. Spronk HM, Soute BA, Schurgers LJ, et al: Matrix Gla protein accumulates at the border of regions of calcification and normal tissue in the media of the arterial vessel wall. Biochem Biophys Res Commun 289: 485-490, 2001.

31. Krauss RM and Kesäniemi YA: Cardiovascular disease and hyperlipidaemia. Curr Opin Lipidol 5: 249-251, 1994. 
32. Golledge J, van Bockxmeer F, Jamrozik K, McCann M and Norman PE: Association between serum lipoproteins and abdominal aortic aneurysm. Am J Cardiol 105: 249-251, 2010

33. Greenow K, Pearce NJ and Ramji DP: The key role of apolipoprotein E in atherosclerosis. J Mol Med 83: 329-342, 2005.

34. Walldius G and Jungner I: The apoB/apoA-I ratio: a strong, new risk factor for cardiovascular disease and a target for lipid-lowering therapy-a review of the evidence. J Intern Med 259: 493-519, 2006.

35. Tailleux A, Duriez P, Fruchart JC and Clavey V: Apolipoprotein A-II, HDL metabolism and atherosclerosis. Atherosclerosis 164 $1-13,2002$.

36. Stan S, Delvin E, Lambert M, Seidman E and Levy E: Apo A-IV: an update on regulation and physiologic functions. Biochim Biophys Acta 1631: 177-187, 2003.

37. Kawakami A and Yoshida M: Apolipoprotein CIII links dyslipidemia with atherosclerosis. J Atheroscler Thromb 16: 6-11, 2009.

38. Sadek M, Hynecek RL, Goldenberg S, Kent KC, Marin ML and Faries PL: Gene expression analysis of a porcine native abdominal aortic aneurysm model. Surgery 144: 252-258, 2008

39. Michel JB, Martin-Ventura JL, Egido J, et al: Novel aspects of the pathogenesis of aneurysms of the abdominal aorta in humans. Cardiovasc Res 90: 18-27, 2011.
40. Stec JJ, Silbershatz H, Tofler GH, et al: Association of fibrinogen with cardiovascular risk factors and cardiovascular disease in the Framingham Offspring Population. Circulation 102: 1634-1638, 2000.

41. McCormick ML, Gavrila D and Weintraub NL: Role of oxidative stress in the pathogenesis of abdominal aortic aneurysms. Arterioscler Thromb Vasc Biol 27: 461-469, 2007.

42. Grote K, Flach I, Luchtefeld M, et al: Mechanical stretch enhances mRNA expression and proenzyme release of matrix metalloproteinase-2 (MMP-2) via NAD(P)H oxidase-derived reactive oxygen species. Circ Res 92: e80-e86, 2003.

43. Dubick MA, Hunter GC, Casey S and Keen CL: Aortic ascorbic acid, trace elements, and superoxide dismutase activity in human aneurysmal and occlusive disease. Proc Soc Exp Biol Med 184: 138-143, 1987.

44. Stephens JW, Bain BC and Humphries SE: Gene-environment interaction and oxidative stress in cardiovascular disease. Atherosclerosis 200: 229-238, 2008.

45. Sukhanov S, Higashi Y, Shai SY, et al: Novel effect of oxidized low-density lipoprotein: cellular ATP depletion via downregulation of glyceraldehyde-3-phosphate dehydrogenase. Circ Res 99: 191-200, 2006. 